九州大学学術情報リポジトリ

Kyushu University Institutional Repository

\title{
Lattice Boltzmann Method for Free Surface Impacting on Vertical Cylinder : A Comparison with Experimental Data
}

Mohd, Nik

Interdisicplinary Graduate School of Engineering Sciences, Kyusuh University

Kamra, Mohamed M.

Interdisciplinary Graduate School of Engineering Sciences, Kyushu University

Sueyoshi, Makoto

Research Institute for Applied Mechanics (RIAM), Kyushu University

$\mathrm{Hu}$, Changhong

Research Institute for Applied Mechanics (RIAM), Kyushu University

https://doi.org/10.5109/1929662

出版情報: Evergreen. 4 (2/3)，pp.28-37，2017-09. Green Asia Education Center バージョン：

権利関係 : 


\title{
Lattice Boltzmann Method for Free Surface Impacting on Vertical Cylinder: A Comparison with Experimental Data
}

\author{
Nik Mohd ${ }^{1}$, Mohamed M. Kamra ${ }^{1}$, Makoto Sueyoshi' ${ }^{2}$, Changhong $\mathrm{Hu}^{2 *}$ \\ ${ }^{1}$ Interdisicplinary Graduate School of Engineering Sciences (IGSES), Kyushu University, Japan \\ ${ }^{2}$ Research Institute for Applied Mechanics (RIAM), Kyushu University, Japan \\ *Author to whom correspondence should be addressed, \\ E-mail: hu@riam.kyushu-u.ac.jp
}

(Received, July 10, 2017; accepted, August 30, 2017).

\begin{abstract}
The purpose of the present research is to study three-dimensional lattice Boltzmann method (LBM) simulation on free surface impact phenomena with the validation on a newly performed dam breaking experiment. Large eddy simulation (LES) is implemented in the LBM to enhance the computational efficiency and to relax the restriction of the computational stability. The LBM method involves a surface-tracking technique with free surface algorithm. In this study, the present numerical simulation is validated by comparing wave front propagation and water level elevation with the experimental measurements. A three dimensional numerical simulation on dam breaking with vertical cylinder obstacles are performed and qualitative comparison with the experimental measurement has been made. Good qualitative agreement between numerical simulations and the experiments has been obtained in terms of free surface development, splash pattern and splash distance. For the square cross section cylinder, the water impacts on the cylinder violently and the flow is directed to the sides of the tank. For the circular cross section cylinder, the water flows smoothly around the cylinder and impacts tank wall violently. The results show that the free surface lattice Boltzmann method is efficient for dealing with complex geometrical problems.
\end{abstract}

Keywords: Free surface flow; Vertical cylinder; Lattice Boltzmann method

\section{Introduction}

Many ocean and coastal engineering applications such as offshore structures, risers and bridge piers have the problems of wave impact flows ${ }^{1}$. Understanding the physical phenomena of the breaking waves impacting on the structures will contribute towards prevention of structural damages. Coastal and offshore structures consisting of vertical cylinders are exposed to highly nonlinear and breaking waves in shallow water region ${ }^{2}$. Vertical cylinder design (square or circular) are usually used as the basis for the fundamental structures ${ }^{2,3,4,5)}$. Computational Fluid Dynamics (CFD) is an efficient tool for such problems and it has become important to validate the accuracy of numerical simulations ${ }^{3,4,6,7,8)}$. The validations can be carried out by performing systematic and comprehensive experimental measurement using dam break facilities.

Literature studies show that several simulation studies were validated by comparison to laboratory experimental data produced by Catherine and Harry Yeh from the University of Washington (Raad and Bidoae ${ }^{7}$; Tso-Ren $\mathrm{Wu}^{4)}$; Gomez-Gesteira and Dalrymple ${ }^{8)}$ ). The experiment for the vertical cylinder contains the force and velocity measurement. Tso-Ren $\mathrm{Wu}^{4)}$ used the laboratory measurements for validation of turbulence models with the linear and nonlinear $k-\varepsilon$ model and compared the predicted forces to the laboratory experiments. GomezGesteira and Dalrymple ${ }^{8)}$ modeled a three-dimensional smoothed particle hydrodynamics by comparing it with the same experimental data, whilst Raad and Bidoae ${ }^{7}$ developed the Eulerian-Lagrangian marker and micro cell (ELMMC) method and verified it using the same experimental data. However, it should be pointed out that in the experiment, high speed and high resolution cameras were not used, which are considered to be essential to capture detailed structures of the free surface and very high speed of the gate removing ${ }^{9,10)}$.

Numerical simulations of free surface impact problem have been carried out by many CFD methods, such as FDM and Finite Volume Method (FVM) ${ }^{11,12)}$. Recently the Lattice Boltzmann Method (LBM) ${ }^{13)}$ is also applied to this problem. The LBM was first developed by McNamara and Zanetti ${ }^{14}$. The development of the LBM started from microscopic models, moving on to mesoscopic kinetic theory and later to recover the macroscopic scale continuity. The LBM equation was later used to simulate fluid flows ${ }^{15)}$. In comparison with the traditional CFD 
methods, LBM has the advantages of dealing with complex boundaries, incorporating microscopic interactions, flexible reproduction of the interface between multiple phases, and easy parallelization of the algorithm ${ }^{16)}$. It also provides an alternative way to solve fluid flows, particularly due to its efficient approach for solving a variety of complex fluid dynamics problems, as in the field of multiphysics ${ }^{17)}$. Multiphase problems have been solved with the LBM starting from the work of Gunstensen et al. (1991) ${ }^{18)}$ who investigated the flow of two immiscible fluid and later this work was improved with a second collision operator to the LBM equation by modelling the dynamics of two fluids with different densities and viscosities ${ }^{19}$.

The present study is based on the single phase freesurface model by Ginzburg and Steiner ${ }^{20,21)}$; Korner et al. 22); Thurey ${ }^{22,24)}$. This method was originally applied to filling process of viscoelastic metal alloys and plastic in expending cavities. To the authors' best knowledge, there are few published works on free surface flow, which is generated by a dam break, impacting on threedimensional square cylinder ${ }^{4,7,8}$, while no study have been found on three-dimensional circular cylindrical obstacles. In this study, three-dimensional numerical simulation of such free surface impact problem by using LBM approach, which has not been well studied, is carried out. To validate our numerical approach, an experiment on dam break is newly conducted by using a high speed / high resolution camera system. The C++ Parallel Lattice Boltzmann Method (Palabos) library is used to carry out the numerical simulations ${ }^{25)}$ A validity test was made by performing grid dependency test of the present numerical method on the case of our previous study ${ }^{26)}$. Then, the LBM simulation results are quantitatively compared with the experimental measurements for the wave front propagation and water level elevation at four different locations. Finally, a three dimensional numerical simulation with vertical cylindrical obstacles are performed and qualitatively compared with the experiment.

\section{Experimental and numerical methods}

\subsection{Experimental Setup}

An improved experiment from previous works on CFD validation ${ }^{9,27)}$ is performed in the Research Institute for Applied Mechanics (RIAM), Kyushu University. In the present study, a new experimental setup with a new gate design have been developed. The design of the bottom gate area is much smaller than that in the previous experiment $^{9,27)}$ in order to reduce the effect of suction force on the dry horizontal bed. In addition, two I-Beam structures are attached on the gate structure in order to increase the structure strength. The wire cable and $40 \mathrm{~kg}$ weighing are used to reduce the pulley friction and increase the gate speed. Figures 1 and 2 illustrate the experimental setup displaying the overall experimental system and the schematic diagram, respectively. The experimental setup mainly consists of a mechanical release system, linear guide system and a prismatic tank. The top lid serves to prevent water from leaving the tank after impact as well as fix the vertical cylinder in-place thus making it perfectly stationary. The bottom of the prismatic tank is fixed to the basement of steel and concrete.

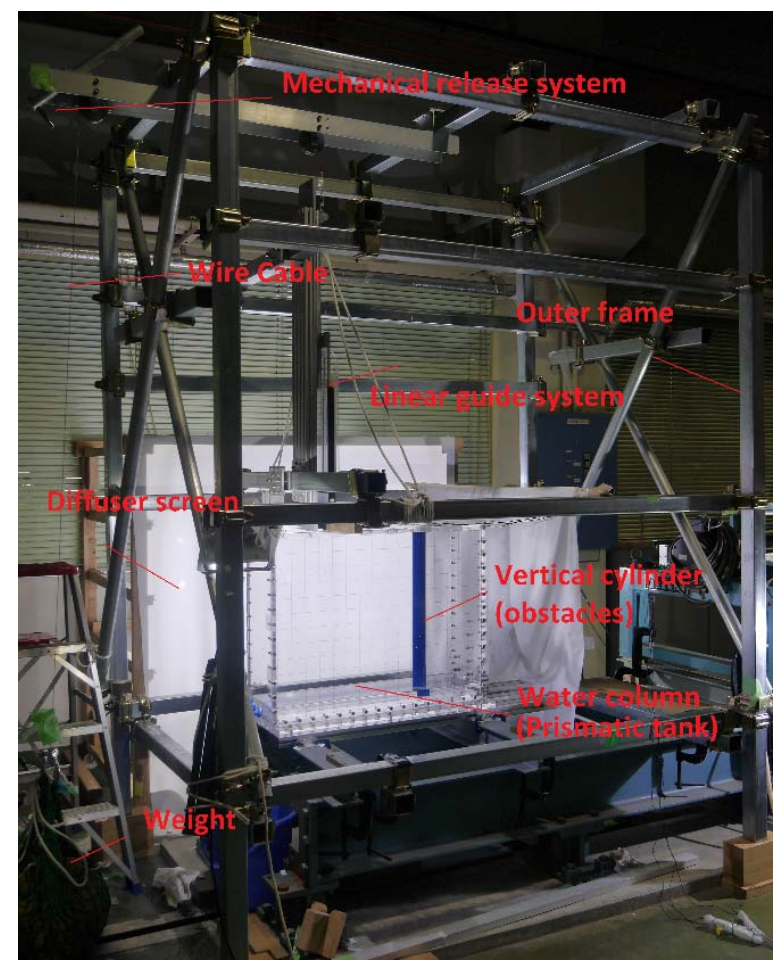

Fig.1: Experimental setup with mechanical and linear guide systems

The schematic diagram depicts two high speed cameras and two personal computers for data recording. The video record is then used for obtaining the free surface profile. Camera 1 is installed at the perpendicular to the measurement area which is used to measure the wave front propagation and the water level elevation. Camera 1 can also be moved to the left side to record the interaction of the free surface with the vertical cylinder from a perspective view. Camera 2 is fixed at a permanent location to measure the initial gate removal movement whilst Camera 1 is located closely to the measurement location of the dam break to measure wave propagation on the right side. Both cameras are used simultaneously. Optical diffuser is connected to the lighting-emitting diode (LED) lighting system to attain a high quality video image. In the experiments, the vertical velocity of gate movement and downstream wave propagation velocity are analysed using a digital motion capturing software (DippMotion PRO). Figure 3 depicts the vertical gate speed movement for two different cases. 

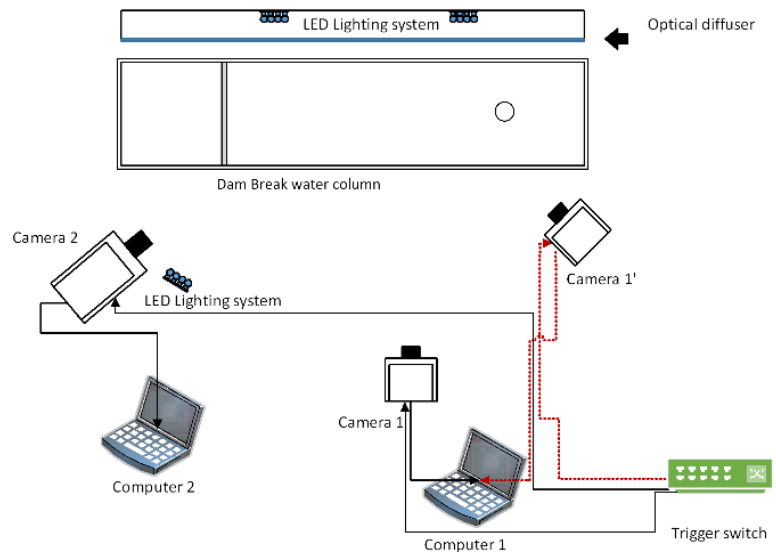

Fig.2: Data acquisition system (DAS) installation

It is found that the gate speeds are inconsistent for different trials which may be caused by several factors; such as the rubber stiffness which may cause friction and the manual control of mechanical release which may cause instability of the weight movement. Such inconsistency were previously reported by $\mathrm{Hu}$ and Sueyoshi ${ }^{9)}$, Lobovsky et al. ${ }^{28)}$ and Zhouteng et al. ${ }^{29)}$

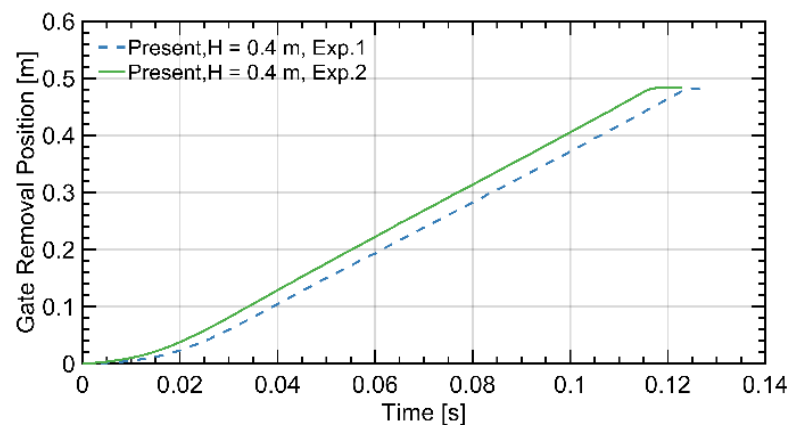

Fig.3: Experimental gate speed duration for $\mathrm{H}=0.4 \mathrm{~m}$.

Water level elevation measurement is another important aspect to be considered in order to understand the free surface phenomena. Water level elevation measurement technique is proposed and the data in four selected locations are discussed. Figures 4 and 5 indicate the details of the selected water level measurement locations and the image processing results, respectively. Four selected locations are labelled as H2 $(0.1 \mathrm{~m}), \mathrm{H} 5(0.3 \mathrm{~m})$, H9 $(0.5 \mathrm{~m})$ and $\mathrm{H} 13(0.7 \mathrm{~m})$. This numbering follows the labelling in Figure 5.

\subsection{Numerical Method}

The lattice Boltzmann method ${ }^{30)}$ provides an alternative approach to the well-established finite-volume, finitedifference and finite-element techniques for solving fluid flows. This method treats the fluid using statistics, simulating the movement and interaction of single particles or ensemble-averaged particle density distributions by solving a velocity discrete Boltzmanntype equation.

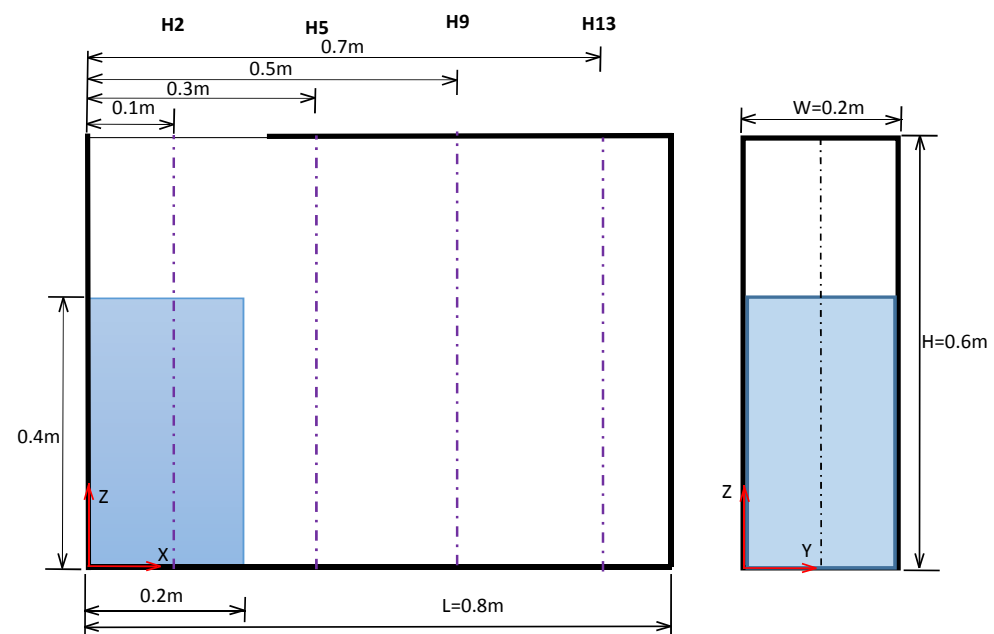

Fig.4: Water level measurement at the selected locations
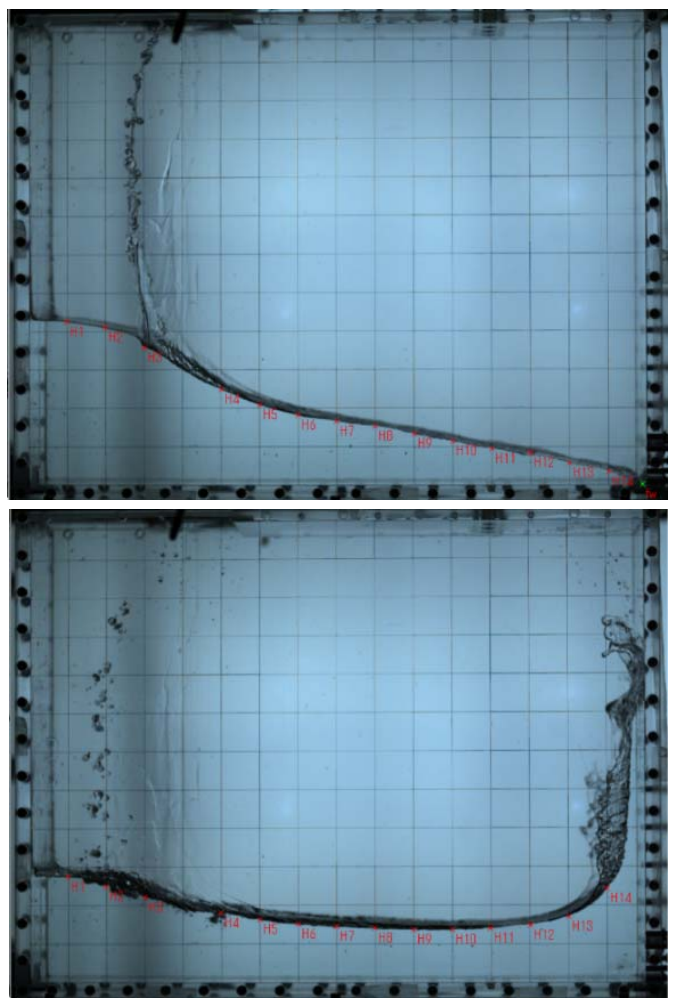

Fig. 5: Water column elevation at the selected frames at $0.299 \mathrm{~s}$ (above) and $0.424 \mathrm{~s}$ (bottom)

\subsubsection{Lattice Boltzmann method}

The LBM with standard three dimensional, nineteen velocities (D3Q19) model for flow field is employed in this work as seen in Figure 6. The LBM allows to describe the dynamics of a fluid by representing the fluid properties in terms of distribution functions. A distribution function $f(x, c, t)$ is a probability density function (PDF) derived from the kinetic theory. The fundamental idea is that 
gases/fluids can be imagined as consisting of a large number of small particles moving with random motions. The exchange of momentum and energy is achieved through particle streaming and billiard-like particle collision.

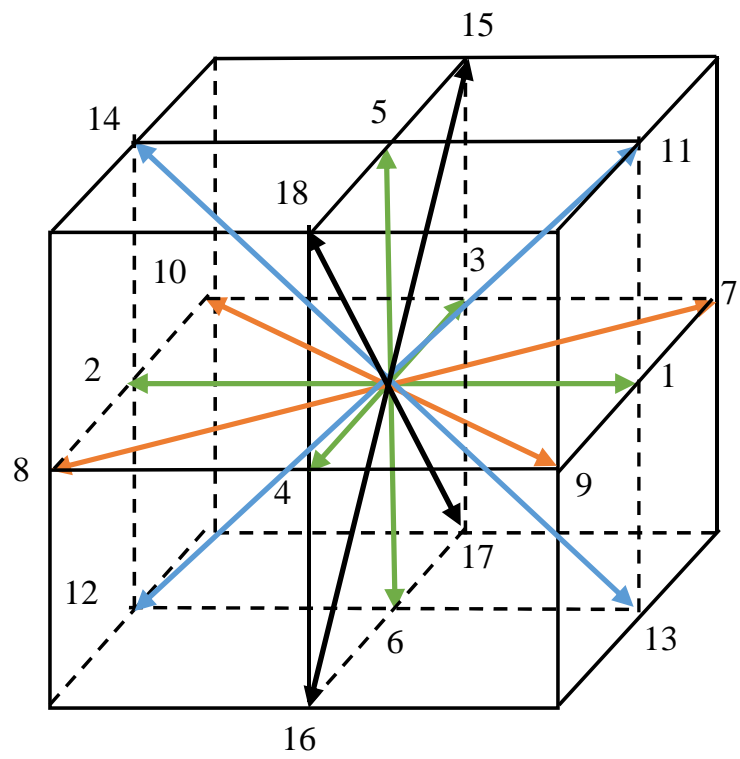

Fig.6: D3Q19 lattice speed for 3D model

This process can be modelled by the Boltzmann transport equation, which is

$$
\frac{\partial f}{\partial t}+c . \nabla f=\Omega(f)
$$

where $f(\boldsymbol{x}, \boldsymbol{c}, t)$ is the particle distribution function, $c$ is the particle velocity and the collision operator, $\Omega$. The LBM simplifies Boltzmann's original idea of gas dynamics by reducing the number of particles and confining them to the nodes of a lattice. The Boltzmann equation can be written for discrete number of velocities ${ }^{11}$ ) as

$$
f_{i}\left(\boldsymbol{x}+\boldsymbol{c}_{\boldsymbol{i}} \Delta t, t+\Delta t\right)-f_{i}(\boldsymbol{x}, t)=\Omega_{i}\left(f_{i}\right)
$$

where the subscript $i$ indicates the direction of changes of the distribution functions. In this study, D3Q19 model is implemented in which D3 is referred to the threedimensional and Q19 for the lattice speed directions. For the collision term, a Bhatnagar-Gross-Kross (BGK) scheme approximation model is used ${ }^{31)}$. This collision is a $1^{\text {st }}$ order model which is implemented in LBM framework.

$$
\Omega_{i}\left(f_{i}\right)=\frac{1}{\tau}\left(f_{i}(x, t)-f_{i}^{e q}(x, t)\right)
$$

where $\tau$ is the e relaxation parameter towards local equilibrium. For simulating single phase flows, it suffices to use (BGK) collision in which equilibrium distribution function is defined as

$$
f_{i}^{e q}(\boldsymbol{x}, t)=w_{i} \rho\left(1+\frac{\boldsymbol{c}_{\boldsymbol{i}} \cdot \boldsymbol{u}}{c_{S}^{2}}+\frac{1}{2} \frac{\left(\boldsymbol{c}_{\boldsymbol{i}} \cdot \boldsymbol{u}\right)}{c_{S}^{4}}-\frac{1}{2} \frac{(\boldsymbol{u})^{2}}{c_{S}^{2}}\right)
$$

where $w_{i}$ are lattice-dependent weighting factors function of a velocity direction. The weighting factors function depends only on macroscopic quantities. The macroscopic density, $\rho$ and velocity, $\boldsymbol{u}$ are defined as the hydrodynamics moments of the distribution functions (DFs) as follows

$$
\begin{aligned}
& \rho=\sum_{i=0}^{18} f_{i} \\
& \boldsymbol{\rho} \boldsymbol{u}=\sum_{i=0}^{18} \boldsymbol{c}_{\boldsymbol{i}} f_{i}
\end{aligned}
$$

\subsubsection{Free surface-tracking technique}

In free surface lattice Boltzmann model, the liquid cells are always being set apart from the gas cell by using a layer of interface cells. The movement of the fluid interface is taken from the mass in the cell, whereby both the mass, $\quad M$ and the fluid fraction, $\varepsilon$ of the cell is defined as follows

$$
M(x, t)=\varepsilon(x, t) \cdot \rho(x, t)
$$

where $\varepsilon(x, t) \in[0,1]$ is the volume fraction. For fluid cells, the mass is equal to their density, the fluid fraction being, $\varepsilon=1$. For empty and boundary cells, the mass exchange need not be considered, as the mass exchange is only calculated according to the streaming step and no DFs are streamed from or into the two latter cell types.

The evolution in time of the fluid mass across the lattice point $\boldsymbol{x}$ is

$$
M(x, t+\Delta t)=M(x, t)+\sum_{i=0}^{18} \Delta M_{i}(x, t)
$$

Similar to the VOF approach in literature ${ }^{20-24,32,33)}$, interface tracking is made by computing the fluxes between cell. For interface cells, a direct calculation of the mass exchange is made from the stream step of the LBM. 


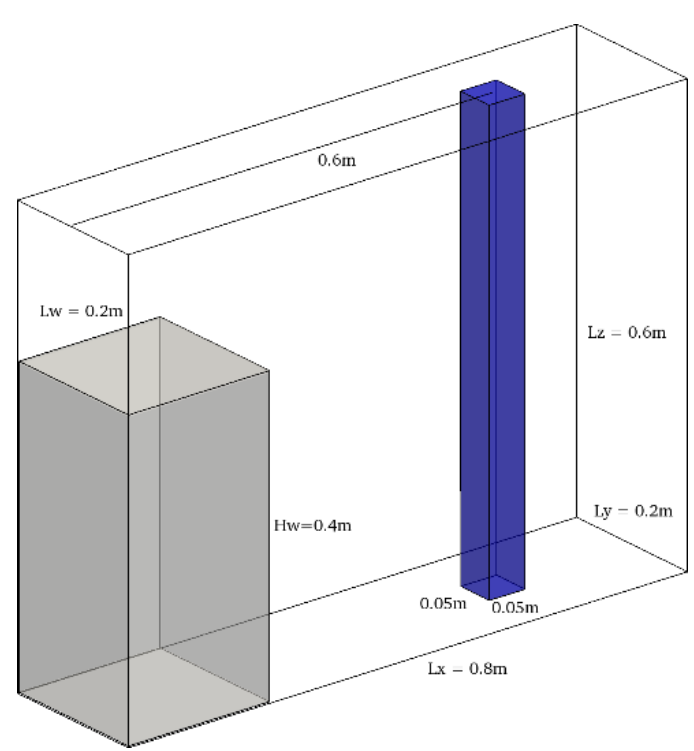

(b) Square cylindrical cross-section

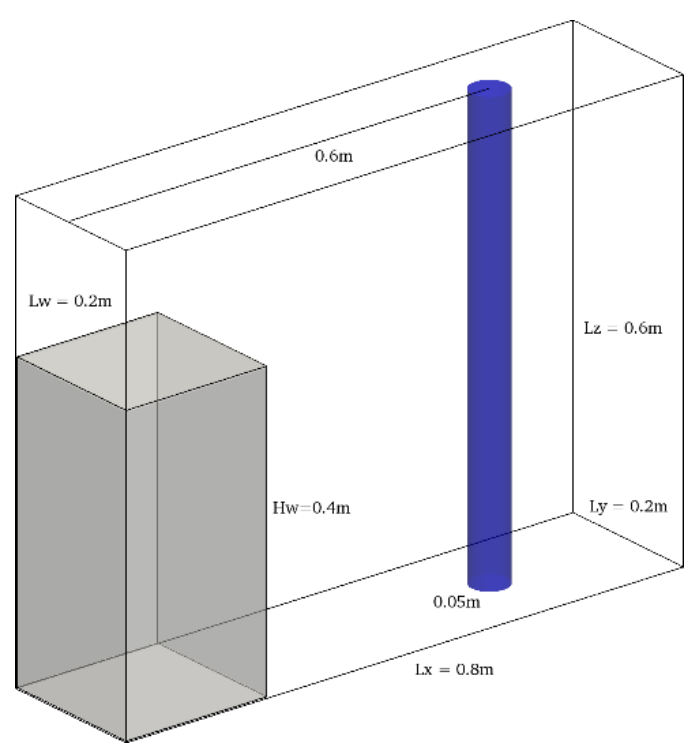

(a) Circular cylindrical cross-section

Fig.7: Dimensions of the vertical cylinder of dam break

For an interface cell at $x$, the mass balance with a neighbor at $\left(\boldsymbol{x}+\boldsymbol{c}_{\boldsymbol{i}} \Delta t\right)$ is specified by

$$
\Delta M_{i}(x, t)=\left\{\begin{array}{c}
0 \\
f_{i}^{\text {out }}\left(\boldsymbol{x}+\boldsymbol{c}_{\boldsymbol{i}} \Delta t, t\right)-f_{i}^{\text {out }}(\boldsymbol{x}, t) \\
\frac{1}{2}\left(\varphi(\boldsymbol{x}, t)+\varphi\left(\boldsymbol{x}+\boldsymbol{c}_{\boldsymbol{i}}\right)\right) \cdot\left(f_{i}^{\text {out }}\left(\boldsymbol{x}+\boldsymbol{c}_{\boldsymbol{i}}\right)\right)-f_{i}^{\text {out }}(\boldsymbol{x}, t)
\end{array}\right.
$$

The volume fraction $\varepsilon(x, t)$ of an interface cell can be computed using Eq. (7) and Eq. (5). On the interface cells thought, not all the PDFs are known. The populations from an empty cell are unknown and need to be reconstructed. The missing distribution functions are reconstructed as follows:

$$
f_{i}^{\text {in }}(\boldsymbol{x}, t)=f_{i}^{e q}\left(\rho^{\text {gas }}, \boldsymbol{u}\right)+f_{\bar{i}}^{e q}\left(\rho^{\text {gas }}, \boldsymbol{u}\right)-f_{i}^{\text {out }}(\mathbf{x}, t)
$$

where $\rho^{g a s}=\rho^{g a s}(\boldsymbol{x}, t)$ and $\boldsymbol{u}=\boldsymbol{u}(\boldsymbol{x}, t)$.

\subsubsection{Boundary conditions}

In the present study, no-slip bounce-back boundary condition is implemented along stationary walls and cylindrical curved surface, of which the numerical geometries are illustrated in Figure 7. This method simply reverses the direction of the distributions which are streamed to the bounce-back nodes ${ }^{34)}$. The bounce-back boundary condition has been shown to be second-order accurate in space when the wall is located at the halfway in between the bounce-back node and the fluid node.

\subsubsection{Smagorinsky Large Eddy Simulation (LES)}

For the free surface flow at high Reynolds number. In order to model such turbulent flow effects at the sub-grid scale, a Large Eddy Simulation (LES) turbulent model is combined with the $\mathrm{LBM}^{35}$. In LES models, the subgridscale eddies are modeled by a subgrid spatial filter and the large spatial-scale turbulent eddy structures which transport the majority of the energy are solved directly. The Smagorinsky model which determines the strain rate tensor with Smagorinsky constant and strain rate tensor through an additional turbulent viscosity can be computed locally by the LBM. For more details on the LBM implementation of the Smagorinsky LES model, the reader is referred to articles ${ }^{24,32,33,35)}$

\section{Results and discussion}

\subsection{Validation on the wave front propagation}

The downstream wave propagation measurement is conducted systematically by using the video motion capturing technique, which is also used by Lobovsky et al. 28). The measurement technique used in the present study utilizes higher resolutions and larger frame-rate imaging as compared with the measurements used in previous studies (Hu \& Sueyoshi ${ }^{9)}$; Kangpi et al. ${ }^{27)}$; Lobovsky et al. ${ }^{28)}$ ) In this study, two measurements of wave fronts are obtained in order to check the repeatability. These measurement results are quantitatively compared and found to agree well with other experiments as shown in Figure 8. It should be noted that in the experimental measurement and numerical simulations, the influence of the three-dimensionality on the wave front could be observed. 


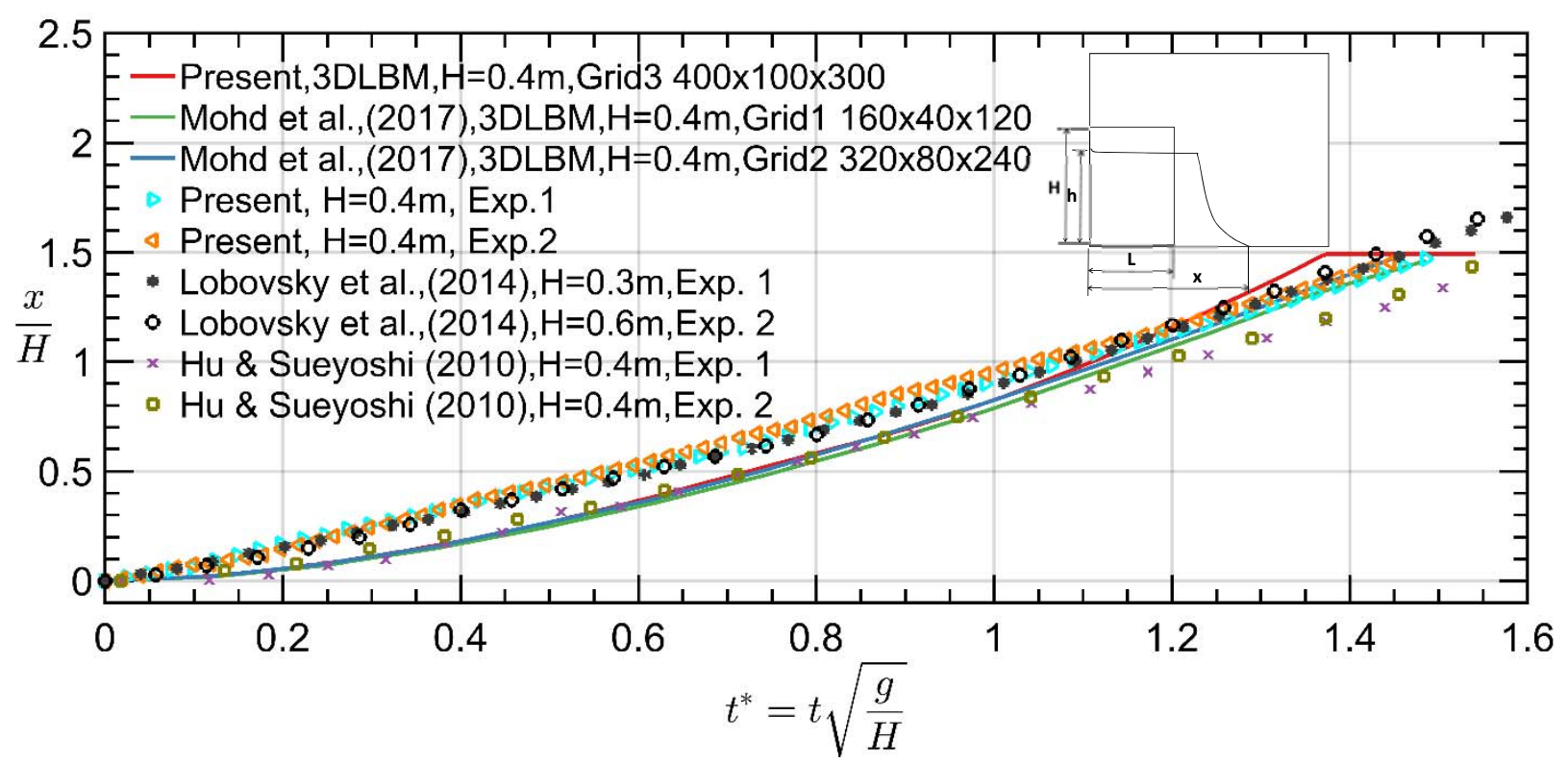

Fig.8: Propagation of wave front from initial gate removal (experiment) until wave arrive first impact compared with numerical solution without gate removal and other data from literature: Hu \& Sueyoshi ${ }^{9)}$, Mohd et al. ${ }^{26)}$, Lobovsky et al. ${ }^{28)}$

In order to check the grid dependency feature of the numerical model, numerical simulations of the dam break problem without obstacles using different grid resolutions are carried out and the results are compared to the experimental measurements in Figure 8. It is shown that although the number of elements has been increased by $20 \%$ grid size of Grid 3 corresponding to lattice spacing of $0.002 \mathrm{~m}$ of which the total lattice node number is 12 million, the results are similar to those with Grid 2 (Mohd et al. ${ }^{26)}$ ) corresponding to lattice spacing of $0.0025 \mathrm{~m}$. Therefore, Grid 2 is selected for further investigation in the subsequent analysis of the study. In the present simulation, after $t^{*}>1.1$, the wave front curve slightly increased until it arrives at the downstream wall. In contrast, the present experimental results are similar to the numerical results by Grid 2 from $t^{*}>1.2$ until it arrives at the downstream wall. Generally, the numerical solutions agree well with the experimental data with some discrepancies which are due to the strong influence of the gate motion for such high water column. As previously noted, in the numerical simulation the gate motion is not modeled.

\subsection{Comparison of water elevations}

Water elevation measurement has been conducted at four different locations from the high speed camera record with high resolutions. The curves presented in Figure 9 provide information about the initial opening of the gate, the water elevation for the downstream flow along the dry surface until the flow reaches the downstream vertical wall. Along with the arrival of the second wave, a strong nonlinear wave appears. Generally, a reasonable agreement is yielded with the results from previous studies despite some discrepancies.

In Figure 9, the numerical results by Grid 2 are selected to be quantitatively compared with the experiment on the water elevation. It is important to note that in the numerical simulation, the effect of the gate is not considered. The water elevation measures are obtained for the locations labeled as $\mathrm{H} 2(0.1 \mathrm{~m}), \mathrm{H} 5(0.3 \mathrm{~m}), \mathrm{H} 9(0.5 \mathrm{~m})$ and $\mathrm{H} 13(0.7 \mathrm{~m})$ as shown in Figures 4 and 5 . The H2 measure is located in the middle of the water column which is measured starting from the top of the free surface profile. On this measure, the results are generally consistent with some discrepancies which may be attributed to the effect of gate resulting in a slower descent ${ }^{29)}$ as well as the arrival of the secondary wave. Such strong secondary wave is observed for $t^{*}>3$ in the experiment which results in a rapid drop of the water elevation, whereas in the numerical simulation the water elevation remains constant. The $\mathrm{H} 5$ and $\mathrm{H} 9$ are measures located after the gate. Based on Figure 9, two observations can be made: the first is that the wave front in the experiment reaches the $\mathrm{H} 5$, and $\mathrm{H} 9$ locations earlier than the numerical simulation. The second is that the rate of increase in the water elevation as well as the maximum water elevation value is higher in the case numerical simulation than the experiment. The first point can be explained by examining the wave front velocity in both cases as shown in Figure 10. In the experiment, at the instant of gate removal the wave front velocity increase rapidly resulting in strong water jet ${ }^{29}$. This is caused by the narrow cross section under the gate and the high head pressure upstream. After the gate is fully open, the wave front velocity becomes nearly constant. This is not case in the numerical simulation as the wave front velocity increases rather steadily until it impacts the 

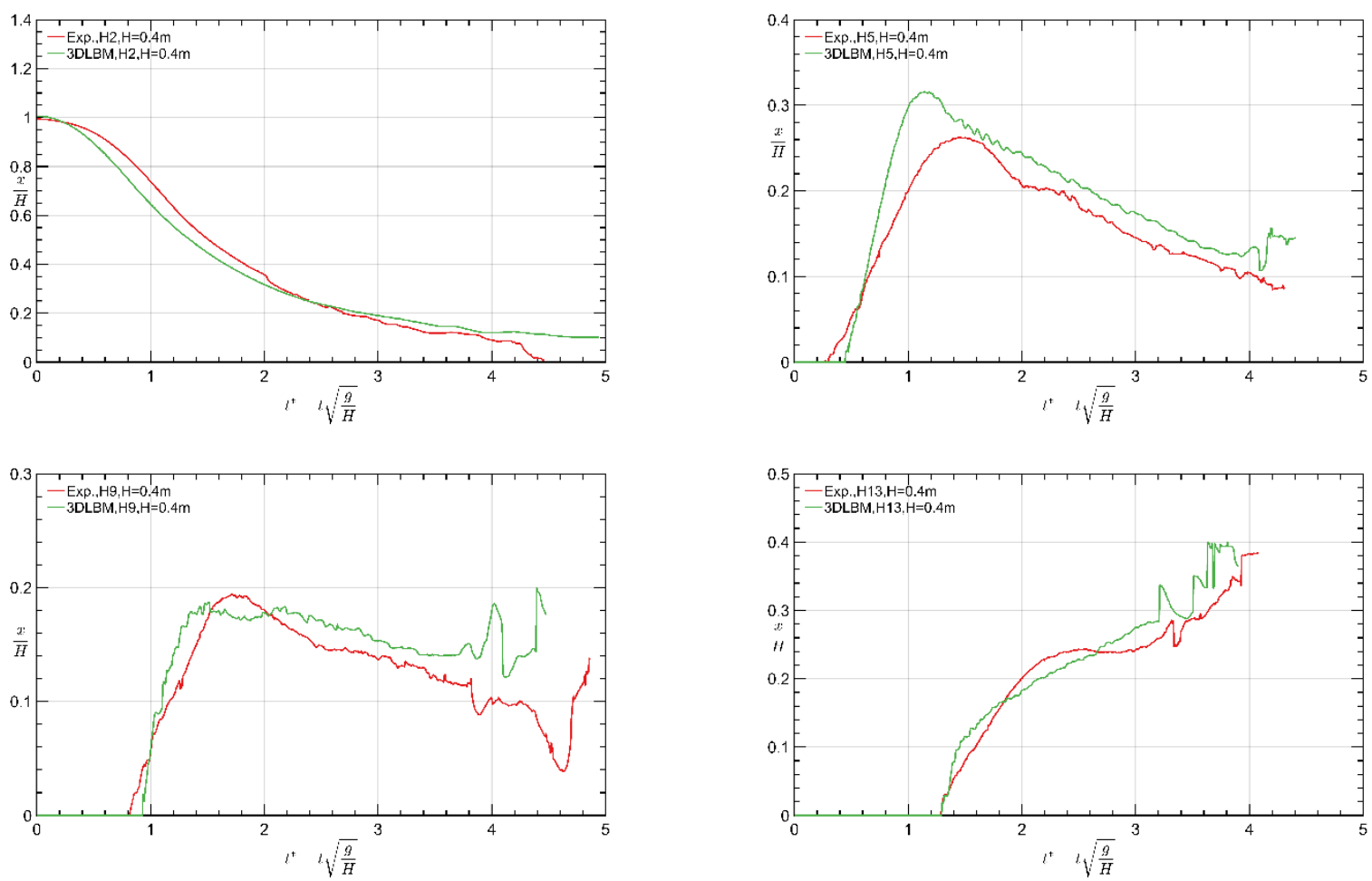

Fig. 9: Water column height elevation at location H2 (top-left), H5 (top-right), H9 (bottom-left) and H13 (bottom-right) for the $\mathrm{H}=0.4 \mathrm{~m}$ water column height compared with the 3DLBM.

vertical wall. As for the second point, this can be explained also by the gate which act as an obstacle restricting the collapse of the water column and resulting in a slower rate of rise in the water level and lower maximum elevation. Further investigation is required for this but it is out of the scope of this study.

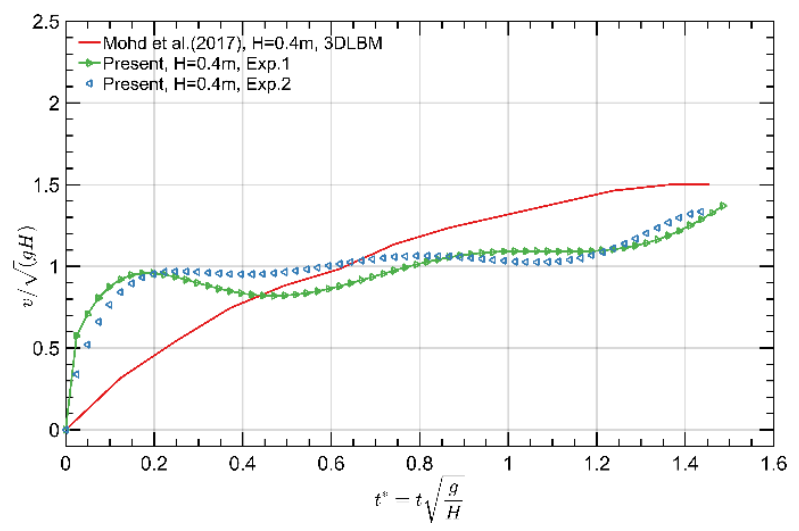

Fig.10: Comparison of wave front velocity with data from a previous study ${ }^{26)}$

The last measure $\mathrm{H} 13$ is located near the downstream vertical wall. At the initial stage, both the wave propagations arrive simultaneously and then the water level rapidly increases until it reaches a maximum position. Later the secondary wave arrives after $t^{*}>3$ producing strong turbulence.

\subsection{Dam Break with vertical cylinder}

A vertical cylinder is placed downstream of the initial water column. The water column collapses under the influence of the gravity and impacts on the cylinder. The dimensions of the liquid column, tank and vertical cylinder is shown in Figure 7. Two cross-sections of the cylinder are considered in this work: a square cross section and a circular cross section.

A series of snapshot of the free surface profile is shown for both cases at selected time steps in Figures 11 and 12. The free surface profiles are compared with the corresponding experiment at the same time instants. From the comparison, the numerical simulation shows good qualitative agreement with the experiment in terms of general shape, obstacle run-up distance, splash pattern and splash distance. A cone-like shaped vortex is observed downstream of the cylinder after the flow impacts on the tank wall. A similar phenomenon is also observed in the experiments. Additionally, for the square cylinder, the water impacts on the cylinder violently and the flow is directed to the sides of the tank. This can be observed at $\mathrm{t}=0.455 \mathrm{~s}$ in Figure 11 and in subsequent snapshots. Consequently, the first impact on the tank wall is much smoother. However, for the circular cylinder, the water flow smoothly, past the cylinder and impacts on the tank wall violently which can be seen at $t=0.455 \mathrm{~s}$ in Figure 12. This shows that the effect of the shape of the cross section on the impact nature is significant. 

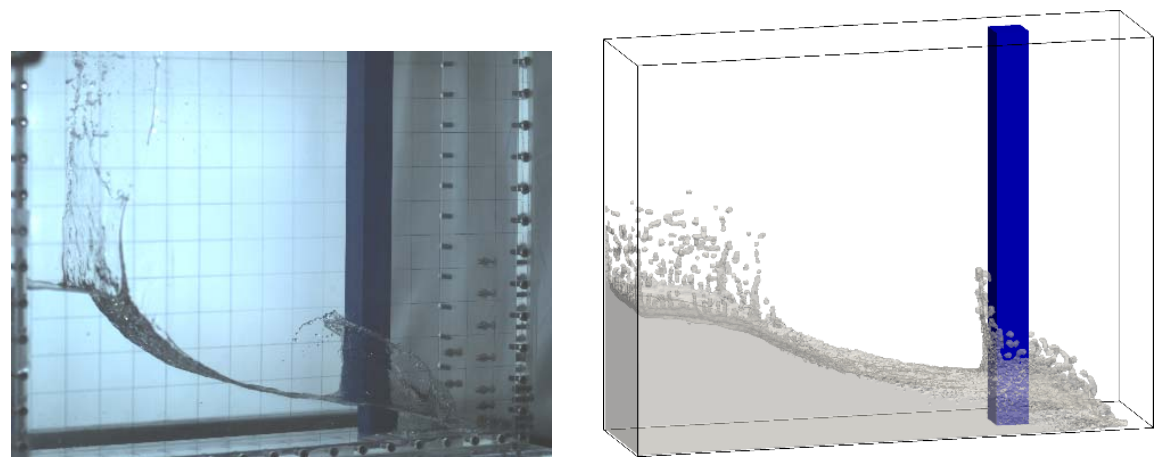

$$
\mathrm{t}=0.275 \mathrm{~s}
$$
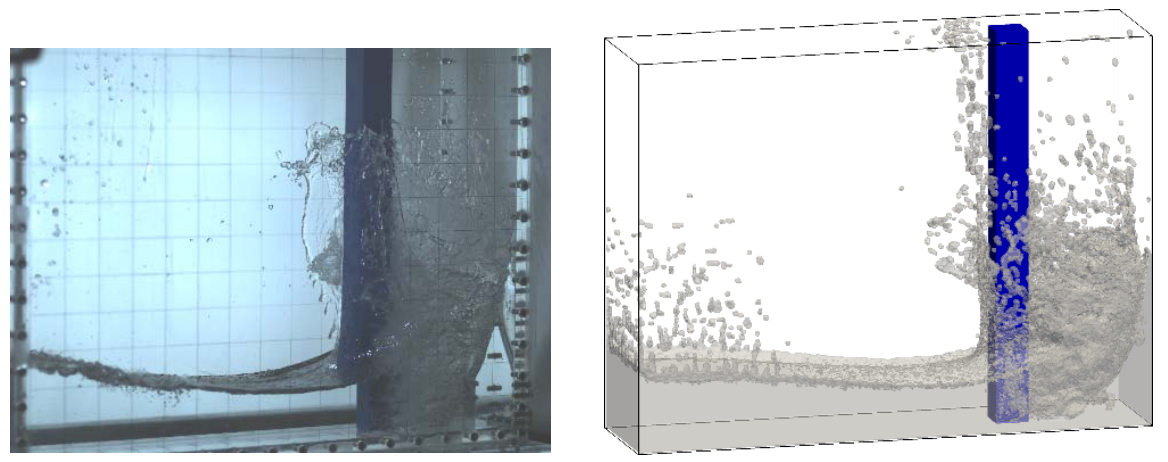

$$
\mathrm{t}=0.455 \mathrm{~s}
$$
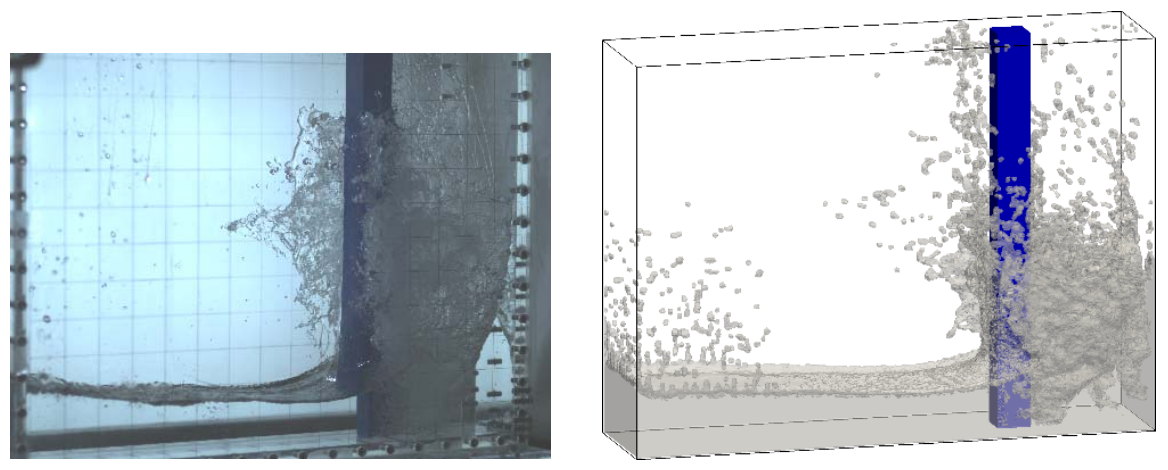

$\mathrm{t}=0.515 \mathrm{~s}$
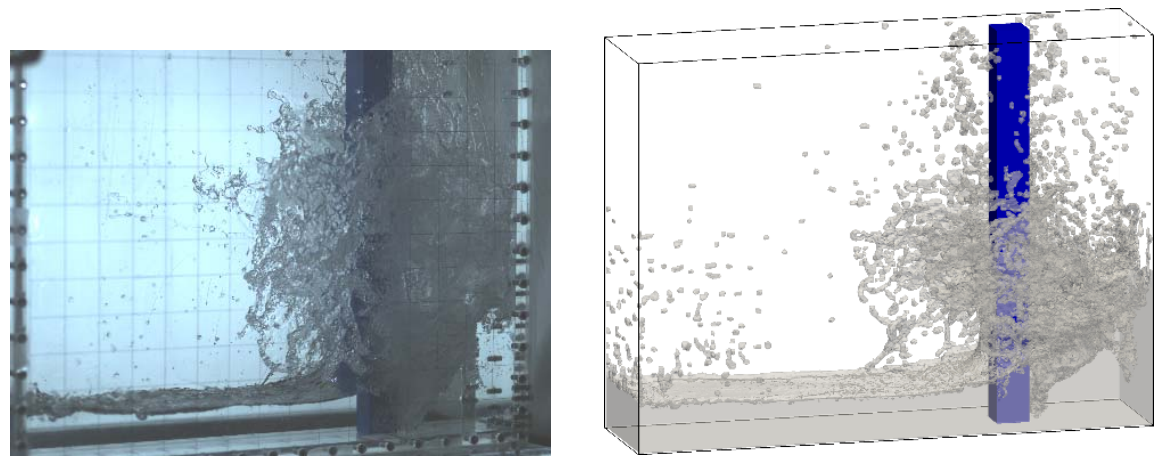

$$
t=0.620 \mathrm{~s}
$$

Fig.11: Snapshots of the free surface for the square cylindrical at selected time steps 

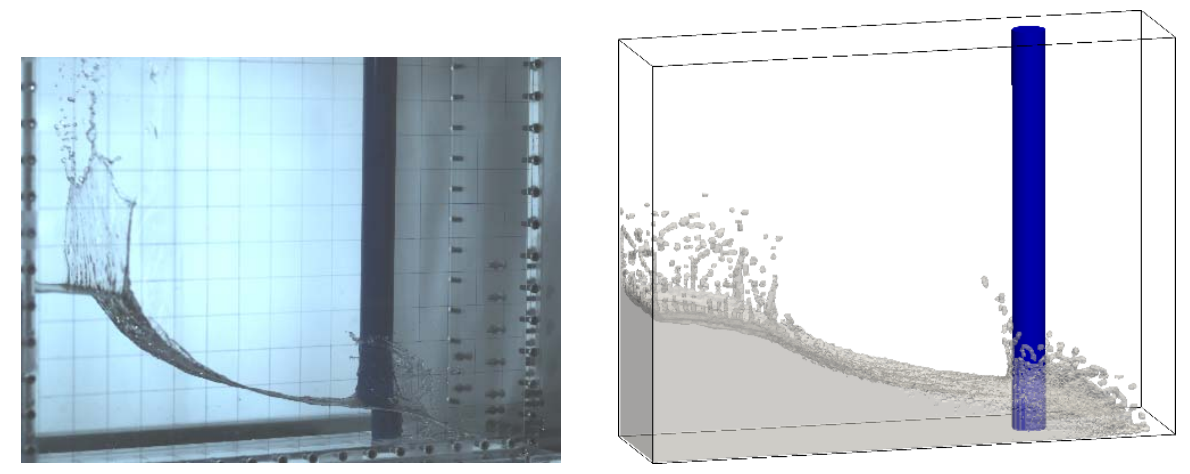

$\mathrm{t}=0.275 \mathrm{~s}$
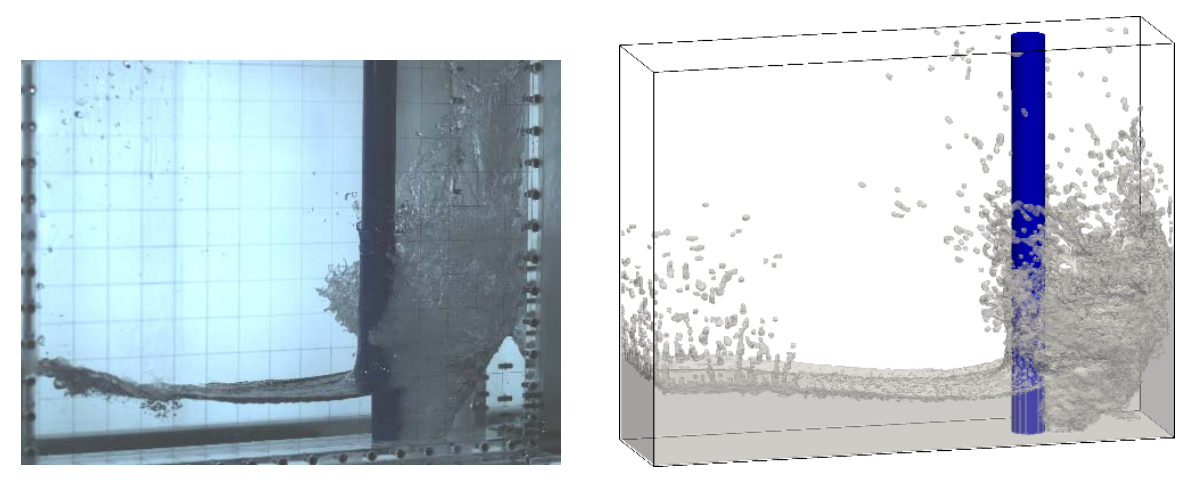

$\mathrm{t}=0.455 \mathrm{~s}$
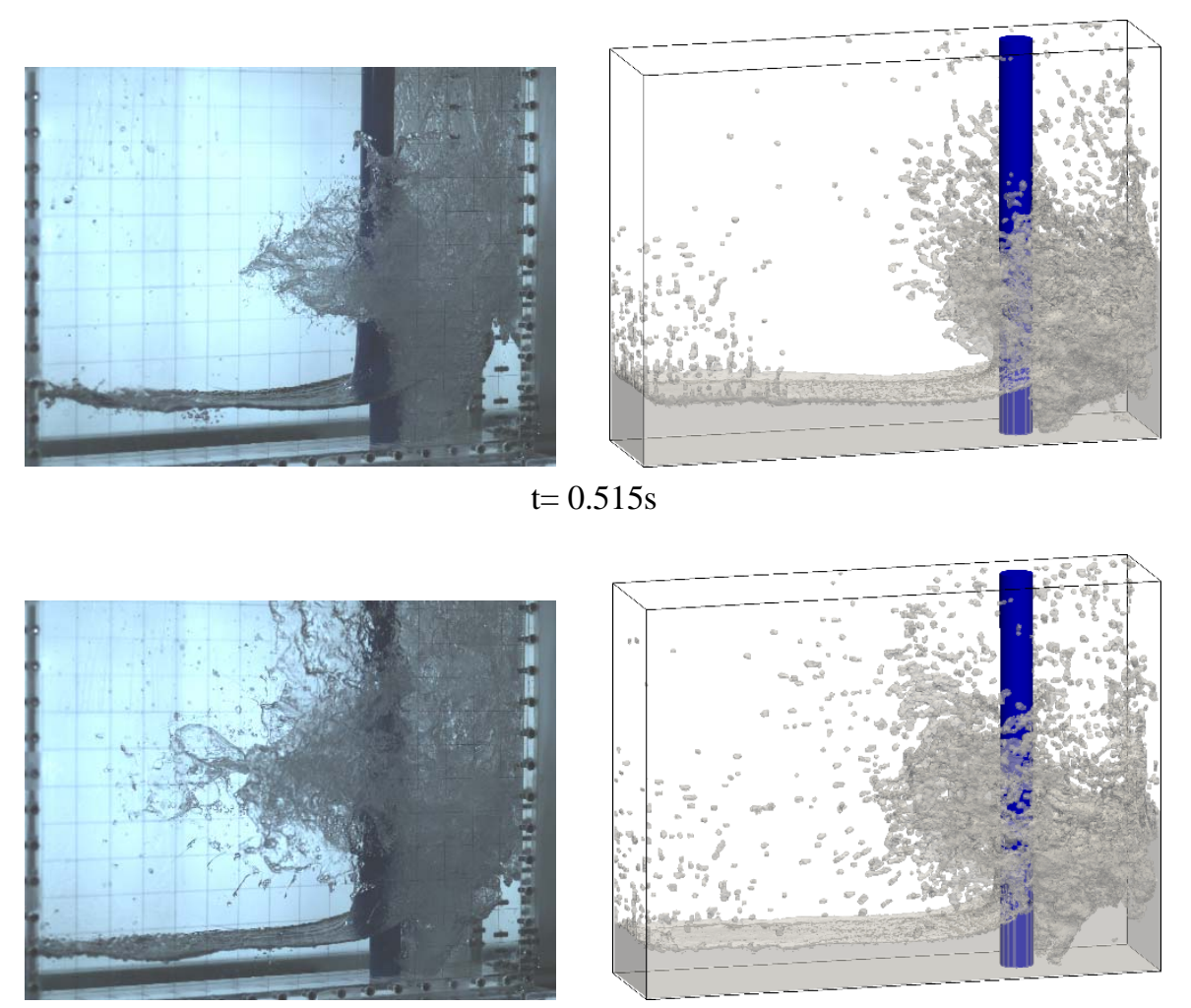

$\mathrm{t}=0.620 \mathrm{~s}$

Fig.12: Snapshots of the free surface for the circular cylindrical at selected time steps 


\section{Conclusion}

The present study aims to study three-dimensional free surface flows by LBM numerical simulation. An experiment on dam break flow impacting on a cylindrical obstacle has been carried out for validation of the LBM approach. The sensitivity of the numerical solution to mesh spacing is investigated by using 3 grid sets. The solution was found mesh independent for mesh spacing less than $0.0025 \mathrm{~m}$. The numerical solutions agree well with the experiment and Grid 2 is selected for the numerical study in this paper through the grid dependency test. Quantitative comparison is carried out between the numerical simulation and the experiment on the water front propagation and the water elevation at four different locations. Reasonably good agreement has been obtained despite some discrepancies due to the influence of the gate motion. A three-dimensional numerical simulation on dam break flow impacting on vertical cylindrical obstacles is conducted and the results are compared with the experiment. A good qualitative agreement between the numerical simulation and the experiment is obtained in terms of general shape, splash pattern and splash distance. This shows that the cross section shape of the cylinder significantly influences the impact phenomena.

\section{Acknowledgement}

The authors would like to thank Ocean Dynamics System laboratory members Akihiro Ohnishi and Kento Obara for their assistance in performing experiments. A deep appreciation to Yann Thorimbert, University of Geneva for constructive discussions on free surface lattice Boltzmann method.

\section{References}

1) Manjula R, Sannasiraj SA, Saravanan $S$. AquaticProcedia 4:227 - 23(2015).

2) Alagan M, Ros X, Bihs H, Myrhaug D. Energy Procedia. 94(1876):443-451(2016).

3) Kamath A, Alagan Chella M, Bihs H, Arntsen ØA. Ocean Eng. 128:105-115(2016).

4) Tso-Ren Wu. Doctoral Thesis, Cornell University, (2004).

5) Qi ZX, Eames I, Johnson ER. J. Fluid Mech. 756,716-727(2014).

6) Kamra MM, Hu C. ISOPE :301-307 (2017).

7) Raad P.E, Bidoae R. Jounral of Computational Physics 203:668-699 (2005).

8) Gomez-Gesteira M, Dalrymple R.A. J.Waterway, Port, Coastal, Ocean Eng. 130:63-69 (2004).
9) Hu C, Sueyoshi M. J Mar Sci Appl. 9(2):109-114 (2010).

10) Martin JC, Moyce WJ. Philos Trans $R$ Soc London Ser A, Math Physys Sci.244(288):325-334(1952).

11)He X, Luo L-S. Phys Rev E. 56(6):6811-6817 (1997).

12) Shan X, Rothman S. flows. Phys Rev E. 47(3)(1993).

13)He X. J.Comput. Phys,134, 315:306-315(1997).

14)Zanetti G, Superiori S. PhysRevLett.61.2332(2015).

15)Succi S. The Lattice Boltzmann Equation. 2001.

16)Jiang F, Hu C. Appl Ocean Res. 45:1-9 (2014).

17)Janßen CF, Grilli ST, Krafczyk M. Comput Math with Appl. 65(2):211-229(2013).

18) Gunstensen AK, Rothman DH. PhysRevA. 43.4320(1991).

19) Grunau D, Chen S, Physics of Fluids 5, 2557 (1993).

20)Ginzburg I, Steiner K I. Ginzburg, K. Steiner Berichte des Fraunhofer ITWM, Nr. 28(2001).

21) Ginzburg I, Steiner K. J Comput Phys. 185(1):6199(2003)

22)Korner, C , Thies M, Hofmann, T, Thurey, $\mathrm{N}$ and Rude U. J Stat Phys. 121:179-196 (2005).

23)Thies M. Diplome Thesis, University of ErlangenNuremberg, Germany (2005).

24) Thurey N. Doctoral Thesis, University of ErlangenNuremberg, Germany (2007).

25) http://www.palabos.org. Accessed October 1, 2016

26) Mohd N, Kamra MM, Sueyoshi M, Hu C. Evergreen 4(1),pp 29-35 (2017).

27)Liao K, Hu C, Sueyoshi M. Appl Ocean Res. 50:192208(2015).

28)Lobovsky L., Botia-Vera E.,Castellana F., Mas-Soler J.,Souto-Iglesias A. J Fluids Struc. 48: 407-434 (2014

29)Zhouteng Y., Xizeng Z., Zhengzhi D. J Mar Sci Technol 21:579-591 (2016).

30)Hasslacher B, Lallemand P. Complex Systems 1 1:649-707(1987).

31)Bhatnagar, P. , Gross, E. , Krook, M. Phys Rev. 94(3):511-525(1954).

32) Parmigiani A, Latt J, Begacem MBEN, Chopard B. Int J Mod Phys C. 24(12):1-8 (2013).

33) Thorimbert Y, Latt J, Cappietti L, Chopard B. Int J Mar Energy. 14:41-51(2016).

34)Lavallee, P.,Boon, J.P., Noullez, A. Physica D., 47:233 (1991).

35)Manfred Krafczyk, Jonas Tolke L-SL. Int J Mod Phys B. 17:33-39 (2003). 